\title{
Effect of Intra-articular Hyaluronic Acid Injection on Hemiplegic Shoulder Pain After Stroke
}

\author{
Myung Hun Jang, $\mathrm{MD}^{1}$, Chang-Hyung Lee, $\mathrm{MD}$, $\mathrm{PhD}^{2}$, Yong-Il Shin, $\mathrm{MD}$, $\mathrm{PhD}^{2}$ \\ Soo-Yeon Kim, MD, $\mathrm{PhD}^{2}$, Sung Chul Huh, $\mathrm{MD}^{2}$ \\ 'Department of Rehabilitation Medicine, Pusan National University Hospital, Pusan National University School of Medicine, \\ Busan; ${ }^{2}$ Department of Rehabilitation Medicine, Pusan National University Yangsan Hospital, \\ Pusan National University School of Medicine, Yangsan, Korea
}

\begin{abstract}
Objective To evaluate the efficacy of intra-articular hyaluronic acid (IAHA) injection for hemiplegic shoulder pain (HSP) after stroke.

Methods Thirty-one patients with HSP and limited range of motion (ROM) without spasticity of upper extremity were recruited. All subjects were randomly allocated to group A $(n=15)$ for three weekly IAHA injection or group B $(n=16)$ for a single intra-articular steroid (IAS) injection. All injections were administered by an expert physician until the 8th week using a posterior ultrasonography-guided approach. Shoulder joint pain was measured using the Wong-Baker Scale (WBS), while passive ROM was measured in the supine position by an expert physician.

Results There were no significant intergroup differences in WBS or ROM at the 8th week. Improvements in forward flexion and external rotation were observed from the 4th week in the IAHA group and the 8th week in the IAS group. Subjects experienced a statistically significant improvement in pain from the 1st week in the IAS and from the 8th week in IAHA group, respectively.

Conclusion IAHA seems to have a less potent ability to reduce movement pain compared to steroid in the early period. However, there was no statistically significant intergroup difference in WBS and ROM improvements at the 8th week. IAHA might be a good alternative to steroid for managing HSP when the use of steroid is limited.
\end{abstract}

Keywords Adhesive capsulitis, Stroke, Hyaluronic acid, Intra-articular injections

\section{INTRODUCTION}

Hemiplegic shoulder pain (HSP) is a very common complication after stroke [1]. Paralysis imposes a condi- tion of immobility that may contribute to the development of glenohumeral joint stiffness which secondarily may cause pain [2]. Diagnosis of HSP typically is based on a combination of clinically signs and symptoms of shoul-

Received March 10, 2016; Accepted May 10, 2016

Corresponding author: Myung Hun Jang

Department of Rehabilitation Medicine, Pusan National University Hospital, 179 Gudeok-ro, Seo-gu, Busan 49241, Korea. Tel: +82-51-240-7485, Fax: +82-51-247-7485, E-mail: zmh1048@naver.com

ORCID: Myung Hun Jang (http://orcid.org/0000-0002-9694-5409); Chang-Hyung Lee (http://orcid.org/0000-0001-6460-6368); Yong-Il Shin (http:// orcid.org/0000-0001-7894-0930); Soo-Yeon Kim (http://orcid.org/0000-0001-5679-0126); Sung Chul Huh (http://orcid.org/0000-0002-7740-8050).

(c) This is an open-access article distributed under the terms of the Creative Commons Attribution Non-Commercial License (http://creativecommons.org/ licenses/by-nc/4.0) which permits unrestricted noncommercial use, distribution, and reproduction in any medium, provided the original work is properly cited. Copyright ( 2016 by Korean Academy of Rehabilitation Medicine 
der pain. However the mechanisms of pain have not been clearly identified, and HSP is difficult to define. It is classified as adhesive capsulitis, shoulder subluxation, complex regional pain syndrome, and central pain according to its causes [3]. After stroke, the primary changes to the hemiplegic shoulder complex include poor positioning of the scapula and the humerus, and immobility. The association found between spasticity, limited range of motion (ROM) and shoulder pain highlights the relationship between the altered biomechanics of the shoulder joint and HSP. For this reason HSP can be defined as a chronic pain syndrome that is consequent to the changes in the musculoskeletal elements of the shoulder complex $[4,5]$. The clinical presentation of HSP mimics that of adhesive capsulitis [6]. Gamble stated that adhesive capsulitis was found significantly more often among hemiplegic patients than the normal population [7]. However, the exact etiology of HSP remains unknown and many factors may be involved. The most common problem is that it interrupts upper limb rehabilitation and daily activities [8].

There have been no specific approaches and management guidelines for HSP patients. Generally, non-surgical treatments of HSP have various options. These may include physical therapies such as positioning of the upper limb, wearing shoulder hemiplegic slings, taking oral pain relievers, applying a moist heating pad, and using of functional electrical stimulation and therapeutic ultrasound. These treatments must be aimed at returning the patients to their daily activities and improving the ROM of the shoulder.

Steroid injections are frequently used for their local anti-inflammatory effect. However, it is well-known that repeated intra-articular steroid (IAS) therapy interferes with cartilage metabolism and may lead to rapid destruction of the joint, resulting in joint arthropathy [9-12]. Nakazawa et al. [13] demonstrated in animal studies that steroid induces chondrocyte apoptosis and arthropathy.

There are many studies investigating the efficacy of hyaluronic acids for pain relief and their effect on the metabolism of articular cartilage, synovial tissue and fluid. Hyaluronic acid might have an anti-inflammatory effect in addition to a regeneration effect since it is a component of cartilage matrix such as glycosaminoglycan and proteoglycan $[14,15]$. Although intra-articular hyaluronic acid (IAHA) injection has shown some promising results in the treatment of patients with adhesive capsulitis, its effect remains controversial.

A previous study in a non-stroke population showed that the effects of IAHA and steroid on adhesive capsulitis were similar [16]. However, there are no previous studies in stroke patients comparing the effects of IAHA versus steroid injection on HSP. The purpose of this study was to compare the efficacy of IAHA with IAS injection on poststroke HSP.

\section{MATERIALS AND METHODS}

\section{Study subjects}

A single-blind, randomized, controlled clinical study comparing the effect of IAHA and IAS on pain and ROM was carried out with post-stroke patients with HSP. The study period was May 2012 to October 2013. The study subjects were patients who were hospitalized at the rehabilitation center of Pusan National University Yangsan Hospital or were presented to the clinic with HSP. The informed consent form was approved by the Institutional Review Board of Pusan National University Yangsan Hospital prior to the study.

The study subjects were 31 patients who developed post-stroke hemiplegia and HSP within the first 3 months after stroke had a limited passive ROM of a capsular pattern on physical examination and had a Wong-Baker Scale (WBS) score of at least 2. Subjects who had mild or no cognitive function impairments with Mini-Mental State Examination above 20 and could accurately reflect the degrees of pain that they experienced during the study were included.

Subjects were excluded if they had previous shoulder diseases such as rheumatoid arthritis or complex regional pain syndrome type 1 on the affected shoulder, a resistance greater than a Modified Ashworth Scale score of 3, previous history of trauma, previous steroid injection, pain or disability of the wrist joint, elbow joint, and metacarpophalangeal joints, or a communication disorder due to aphasia. If multiple combinations of drugs were used to alleviate patient's pain, those cases were also excluded from the study.

Physical examination and ultrasonography (US) were performed to evaluate HSP. One of the authors who is a board certified physiatrist (Lee) with 10 years of experience in ultrasonographic evaluation and musculoskeletal rehabilitation, conducted the manual muscle testing 
of the rotator cuff, and evaluated the degree of pain of the affected shoulder including the possibility of shoulder impingement syndrome using Neer, Hawkins, and Yocum tests. The study subjects underwent US by the same physiatrist to check for rotator cuff impingement syndrome and tendonitis, full thickness rotator cuff tear, calcification of the shoulder, and abnormality of subacromial bursa and biceps, and they were excluded if they had any abnormalities. In addition, patients who were recently taking anti-platelet or anticoagulant agents stopped these medications 5 days prior to the injection to prevent injection-induced hemorrhage.

\section{Study methods}

A total of 31 subjects randomized into two groups, underwent an US-guided intra-articular injection into the shoulder with the following medications:

Group A=0.5\% lidocaine $4 \mathrm{~mL}+$ high molecular weight sodium hyaluronate $2 \mathrm{~mL}$

Group $\mathrm{B}=0.5 \%$ lidocaine $4 \mathrm{~mL}+$ triamcinolone acetate $40 \mathrm{mg} / \mathrm{mL}+$ isotonic saline $1 \mathrm{~mL}$

With patients in a sitting position the injections were performed using an US-guided posterior approach at the exact injection site confirmed by expansion of the shoulder joint capsule. The patient was in a sitting position with the ipsilateral hand crossing the chest. The scapular spine was palpated, and the ultrasound probe was placed just caudal and parallel to the lateral end of the spine. A 22-gauge needle was inserted in-plane from the lateral aspect of the probe and directed between the edge of the labrum and the humeral head [17]. To limit the effects of oral pain relievers, single dose of NSAIDs was given to both groups. Patients in group A received three injections weekly, while patients in group B received a single injection in the 1st week. Moreover, the injection volume used in each group was equally $6 \mathrm{~mL}$ to prevent any effect of hydrostatic pressure expansion and to focus only on the pharmacological effects of the hyaluronic acid and steroids. All patients underwent 1 hour of physical therapy 5 times per week for 3 weeks during the hospitalization period. Physical therapy such as active and passive joint movement with transcutaneous electrical nerve stimulation therapy was performed, and the physical therapists were educated about the physical therapy program before the study began in order to standardize the therapeutic program.

The assessment of therapeutic effects was performed prior to the start of the study, and in the 1st, 4th, and 8th weeks. WBS scores were evaluated at night (night pain) and during the day (movement pain). WBS is a 6-item horizontal scale with 6 facial expressions for clinical pain assessment. The first face represents 'no pain' and the 6th face represents 'the worst possible pain. Patients were asked to mark the face that expresses their level of pain. Face figures are scored between 0 and 10, the least score representing 'no pain' [18]. The passive shoulder ROM was evaluated using a goniometer in every direction except adduction and internal rotation in the supine position. The degrees of shoulder flexion and abduction were measured with the elbow in extension in supine position. The ROM of external rotation was evaluated with $90^{\circ}$ of shoulder abduction and elbow flexion was evaluated with the arm in the side position. In order to measure the exact ROM, the joint should be positioned and the proximal segment stabilized, thereby isolating the articular movement being evaluated. The passive ROM was measured by the examiner moving each subject's arm until limited mechanically or by pain. The physiatrist who carried out the initial assessment and performed the intra-articular injection did not participate in follow-up assessments. Follow -assessments were conducted by clinical examiners with over 2 years of experience in the treatment of HSP patients.

The patients were randomized into two groups: group A received an IAHA injection followed by physical therapy, while group B received an IAS injection followed by physical therapy. Randomization was performed using the block randomization method prior to the clinical trial.

\section{Statistical analysis}

Sample size was calculated with statistical power analysis program G*Power ver. 3.1 (http://www.gpower. hhu.de/). For alpha of 0.05 (2-tailed test), $80 \%$ power, 2 groups, 35 subjects were required. A total of 40 subjects would be necessary to allow for $15 \%$ loss to follow-up.

In this study, subjects that provided baseline and at least 1 post-injection assessment constituted the intention-to-treat (ITT) analysis, whereas those who completed all follow-up assessment from baseline to the 8th week constituted the per protocol (PP) analysis. For the 
ITT analysis, outcome measurements were analyzed using the last observation carried forward (LOCF) method.

Variations in ROM and WBS after injection treatment of the two groups were compared using the Mann-Whitney $\mathrm{U}$ test. Statistical significance was analyzed using the Friedman test to assess the changes over time in each group. The Wilcoxon signed-rank test was performed as a post-hoc test in cases of a significant difference. Measurements were repeated three times with the mean values being used in analysis. Statistical significance was defined at $p$-values $<0.016$ using Bonferroni correction. The statistical program SPSS ver. 18.0 (SPSS Inc., Chicago, IL, USA) was used for the statistical analysis.

\section{RESULTS}

\section{Patient demographics}

A schematic of the study is shown in Fig. 1. A total of 62 subjects were recruited. Twenty-three of, these had shoulder pathology confirmed by US and physical examination such as full thickness rotator cuff tear, calcification of the shoulder, subacromial bursitis and were excluded. After the first follow-up assessment (1st week after the injection), 8 subjects were lost because of admission to other hospitals, poor general condition or they refused follow-up assessments (Fig. 1). No significant side effects were observed in either group over the 8-week followup period. The mean age of the study subjects in group A was $56.6 \pm 11.3$ years, while that of those in group $B$ was $60.8 \pm 13.7$ years. In group A, $11(52 \%)$ patients had cerebral hemorrhage, while 10 (48\%) had cerebral infarction; in group B, 8 (44\%) had cerebral hemorrhage and 10 (56\%) had cerebral infarction. At baseline, there was no significant difference between the group A and group B in age, sex, stroke type, and duration of stroke (Table 1).

\section{Change in the passive ROM of the shoulder \\ Intention-to-treatment analysis using the LOCH method}

The 39 subjects who had follow-up assessments at least once were included in the ITT analysis. At baseline, the passive flexion of the shoulder was $112.1^{\circ} \pm 28.53^{\circ}$ in group A and $122.2^{\circ} \pm 32.00^{\circ}$ in group B while, the passive external rotation of the shoulder prior to injection treatment was $38.6^{\circ} \pm 24.65^{\circ}$ in group $A$ and $27.5^{\circ} \pm 23.02^{\circ}$ in group $B$.

In the group $A$, passive ROM of the shoulder showed significant improvement of flexion $(\mathrm{p}=0.006$ at 4 th week,

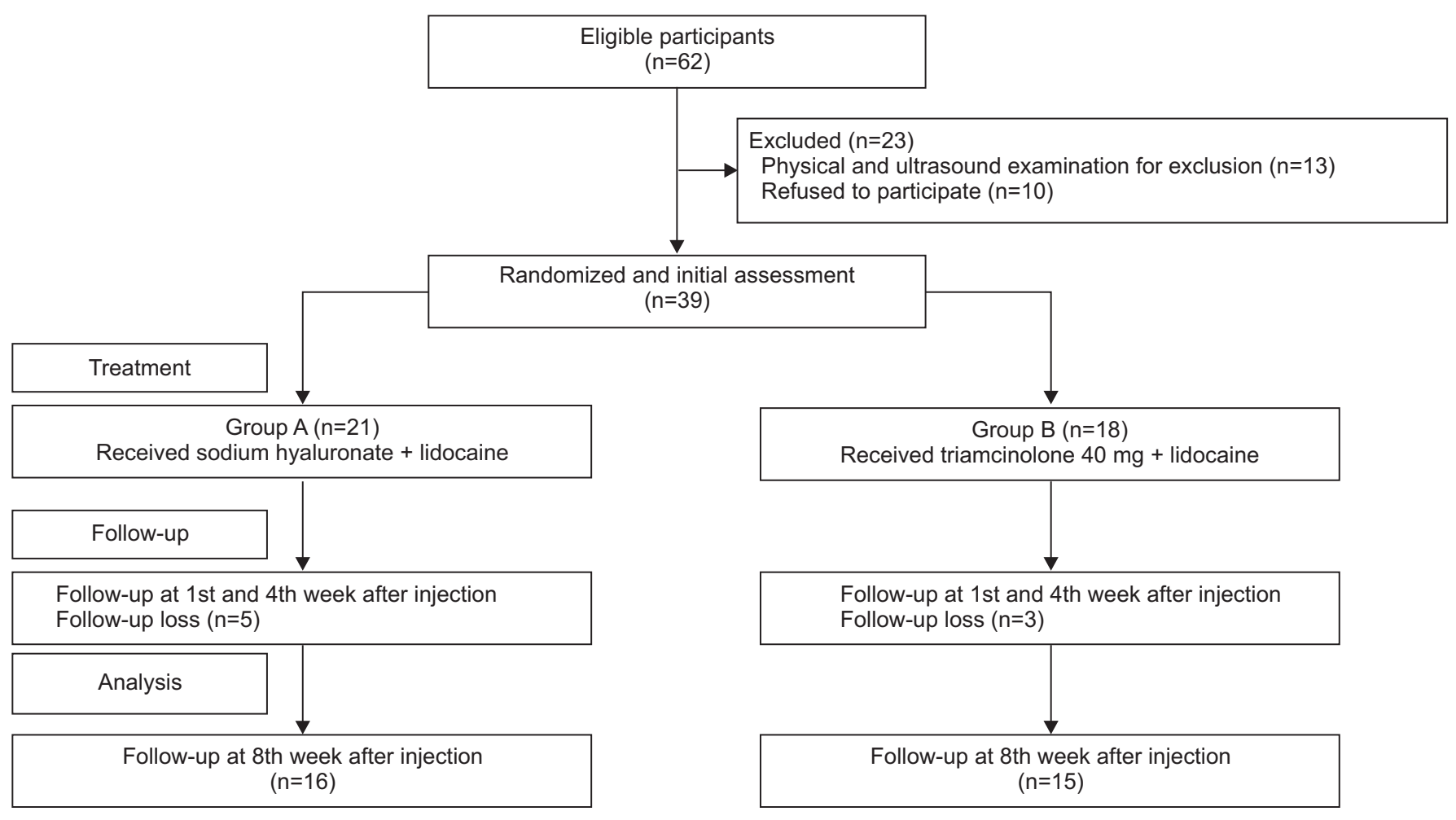

Fig. 1. Participant flowchart of the study. 
Table 1. Basic characteristics at baseline

\begin{tabular}{|c|c|c|c|}
\hline & Group A (hyaluronate) & Group B (steroid) & p-value \\
\hline No. of patients & 21 & 18 & \\
\hline Age (yr) & $56.6 \pm 11.3$ & $60.8 \pm 13.7$ & $0.305^{\mathrm{a})}$ \\
\hline Sex (male:female) & $13: 8$ & $14: 4$ & $0.284^{\mathrm{b})}$ \\
\hline Types of stroke & & & $0.621^{b)}$ \\
\hline Cerebral infarction & $10(48)$ & $10(56)$ & \\
\hline Hemorrhage & $11(52)$ & $8(44)$ & \\
\hline Duration of stroke (day) & $56.76 \pm 29.8$ & $52.94 \pm 33.1$ & $0.707^{\mathrm{a})}$ \\
\hline Hemiplegic side (dominant:non-dominant) & $16: 5$ & 8:10 & $0.042^{b)}$ \\
\hline MRC of hemiplegic side & & & $0.030^{b)}$ \\
\hline$<3$ & 19 & 11 & \\
\hline$\geq 3$ & 2 & 7 & \\
\hline
\end{tabular}

Values are presented as mean \pm standard deviation or number (\%).

MRC, Medical Research Council.

${ }^{a)}$ t-test for between-group comparison, b) chi-square test for between-group comparison.

Table 2. Improvement in passive ROM and WBS at follow-up (ITT analysis with LOCF method)

\begin{tabular}{|c|c|c|c|c|c|c|}
\hline & & & $\begin{array}{c}\text { Group A } \\
\text { (hyaluronate) }\end{array}$ & $\begin{array}{c}\text { p-value } \\
\text { Time effect }\end{array}$ & $\begin{array}{l}\text { Group B } \\
\text { (steroid) }\end{array}$ & $\begin{array}{c}\text { p-value } \\
\text { Time effect }\end{array}$ \\
\hline \multirow[t]{12}{*}{ Passive ROM } & Baseline & Flexion & $122.1 \pm 28.53$ & & $122.2 \pm 32.00$ & \\
\hline & & Abduction & $95.7 \pm 33.03$ & & $82.8 \pm 20.45$ & \\
\hline & & External rotation & $38.5 \pm 24.65$ & & $27.5 \pm 23.02$ & \\
\hline & 1st week & Flexion & $124.3 \pm 27.76$ & 0.248 & $126.7 \pm 35.15$ & 0.245 \\
\hline & & Abduction & $91.4 \pm 29.33$ & 0.176 & $87.22 \pm 19.00$ & 0.246 \\
\hline & & External rotation & $39.0 \pm 25.03$ & 0.680 & $30.6 \pm 22.42$ & 0.288 \\
\hline & 4 th week & Flexion & $133.3 \pm 29.21$ & $0.006^{*}$ & $131.4 \pm 36.45$ & 0.041 \\
\hline & & Abduction & $95.0 \pm 30.78$ & 0.944 & $88.7 \pm 19.20$ & 0.083 \\
\hline & & External rotation & $47.1 \pm 24.42$ & $0.006^{*}$ & $38.9 \pm 24.10$ & 0.018 \\
\hline & 8th week & Flexion & $134.3 \pm 28.39$ & $0.003^{*}$ & $136.1 \pm 36.20$ & $0.014^{*}$ \\
\hline & & Abduction & $92.1 \pm 34.00$ & 0.552 & $89.7 \pm 16.49$ & 0.017 \\
\hline & & External rotation & $50.0 \pm 25.79$ & $0.003^{*}$ & $41.9 \pm 23.90$ & $0.004^{*}$ \\
\hline \multirow[t]{8}{*}{ WBS } & Baseline & Night & $3.8 \pm 2.74$ & & $2.2 \pm 2.41$ & \\
\hline & & Movement & $5.9 \pm 1.64$ & & $6.5 \pm 1.72$ & \\
\hline & 1st week & Night & $3.5 \pm 2.42$ & 0.498 & $1.9 \pm 2.15$ & 0.129 \\
\hline & & Movement & $5.4 \pm 2.31$ & 0.105 & $5.1 \pm 1.59$ & $0.001^{*}$ \\
\hline & 4 th week & Night & $2.3 \pm 2.24$ & $0.003^{*}$ & $1.8 \pm 1.98$ & 0.070 \\
\hline & & Movement & $3.9 \pm 2.13$ & $0.001^{*}$ & $4.1 \pm 2.25$ & $0.000^{*}$ \\
\hline & 8th week & Night & $2.2 \pm 2.17$ & $0.002^{*}$ & $1.8 \pm 1.89$ & 0.143 \\
\hline & & Movement & $3.8 \pm 1.97$ & $0.000^{*}$ & $4.0 \pm 2.09$ & $0.000 *$ \\
\hline
\end{tabular}

Values are presented as mean \pm standard deviation.

ROM, range of motion; WBS, Wong-Baker Scale; ITT, intention-to-treat; LOCF, last observation carried forward. ${ }^{*} \mathrm{p}<0.016$, Wilcoxon signed-rank test. 
$\mathrm{p}=0.003$ at 8 th week) and external rotation ( $\mathrm{p}=0.006$ at 4 th week, $\mathrm{p}=0.003$ at 8 th week) after 4 th week post-injection. In the group $\mathrm{B}$, passive ROM of flexion $(\mathrm{p}=0.014)$ and external rotation $(p=0.004)$ showed significant improvement only at 8th week (Table 2). However, post-hoc testing showed no significant difference between the two groups over 8 weeks ( $p>0.05)$.

\section{Per protocol analysis}

The 31 subjects who completed 8 weeks follow-up assessments were included in the PP analysis. The passive flexion of the shoulder prior to injection treatment was $117.2^{\circ} \pm 27.20^{\circ}$ in group A and $124.0^{\circ} \pm 34.08^{\circ}$ in group B while, the passive external rotation of the shoulder prior to injection treatment was $32.8^{\circ} \pm 24.90^{\circ}$ in group A and $27.3^{\circ} \pm 24.49^{\circ}$ in group $B$.

In group A, passive ROM of the shoulder showed significant improvement of flexion $(\mathrm{p}=0.007$ at 4 th week, $\mathrm{p}=0.004$ at 8 th week), external rotation ( $\mathrm{p}=0.007$ at 4 th week, $\mathrm{p}=0.010$ at 8 th week) after 4 th week post-injection. In the group B, passive ROM of shoulder showed significant improvement of external rotation $(\mathrm{p}=0.010)$ only at the 8 th week. The improvement in passive ROM of the shoulder was greater in the group A at 4th week than in the group B. There was statistically significant improvement at the 8th week in both groups (Fig. 2). However, post hoc testing showed no significant difference be- tween the two groups after 8 weeks ( $\mathrm{p}=0.299$ in flexion, $\mathrm{p}=0.861$ in external rotation at 8 th week).

\section{Change in shoulder pain and WBS scores}

Intention-to-treatment analysis using the LOCH method

The WBS score for night pain prior to injection treatment was $3.8 \pm 2.74$ in group $A$ and $2.2 \pm 2.41$ in group $B$, and movement pain was $5.9 \pm 1.64$ in group $A$ and $6.5 \pm 1.72$ in group $B$.

In group $A$, both night pain $(\mathrm{p}=0.003$ at 4 th week, $\mathrm{p}=0.002$ at 8 th week) and movement pain ( $\mathrm{p}=0.001$ at 4 th week, $\mathrm{p}=0.000$ at 8 th week) were significantly decreased. In the group $\mathrm{B}$, only movement pain was significantly decreased from 1 st week post-injection ( $p=0.001$ at 1 st week, $p=0.000$ at 4 th and 8 th weeks). However, there was no significant difference between two groups for either night pain or movement pain over the time.

\section{Per protocol analysis}

In the group $\mathrm{A}$, both night pain ( $\mathrm{p}=0.011$ at 4 th week, $\mathrm{p}=0.007$ at 8 th week) and movement pain ( $\mathrm{p}=0.006$ at 4 th week, $p=0.001$ at 8 th week) significantly decreased after 4 th week post-injection. In the group B, only movement pain was significantly decreased 1 week after injection and thereafter $(\mathrm{p}=0.002$ at 1 th week, $\mathrm{p}=0.001$ at 4 th and 8th weeks). However, there was no significant difference between groups for either night pain $(\mathrm{p}=0.740$ at 8 th
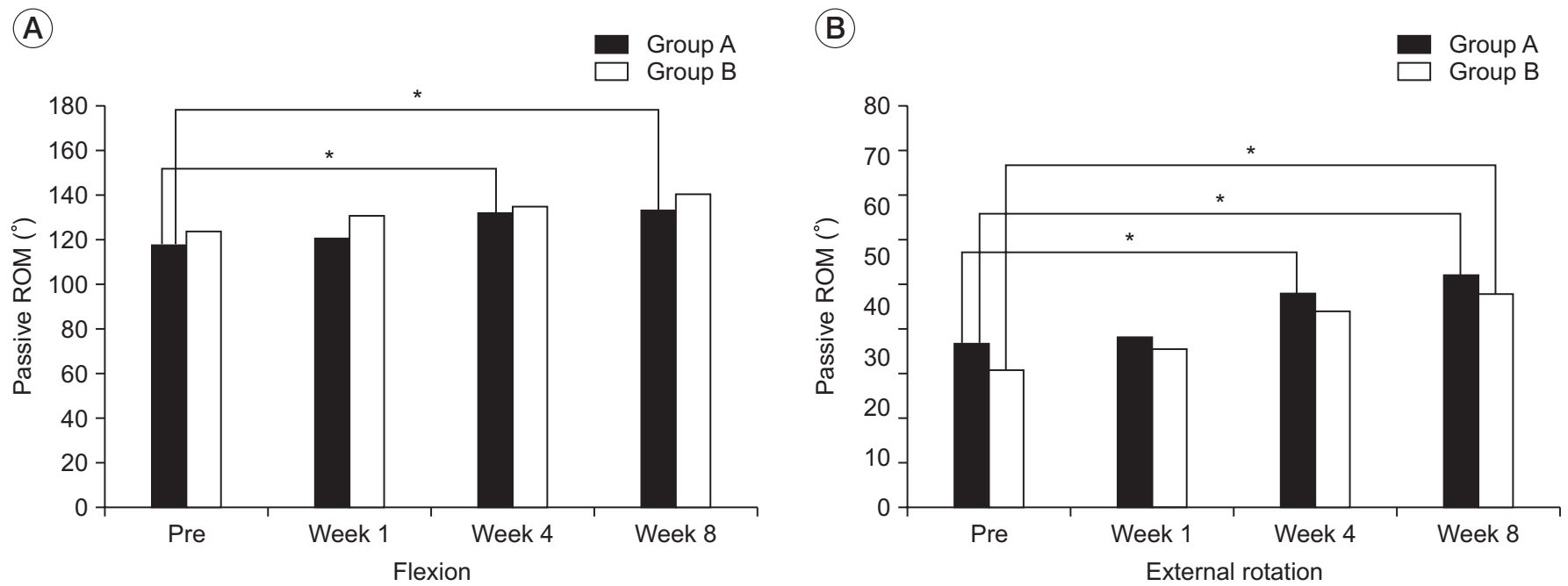

Fig. 2. Passive range of motion improvement at 1,4 , and 8 weeks after the intra-articular injection in the two groups (PP analysis, $\left.{ }^{*} \mathrm{p}<0.016\right)$. Post hoc testing showed no statistical differences between the two groups over time $(\mathrm{p}>0.05)$. ROM, range of motion; WBS, Wong-Baker Scale; PP analysis, per protocol analysis. 

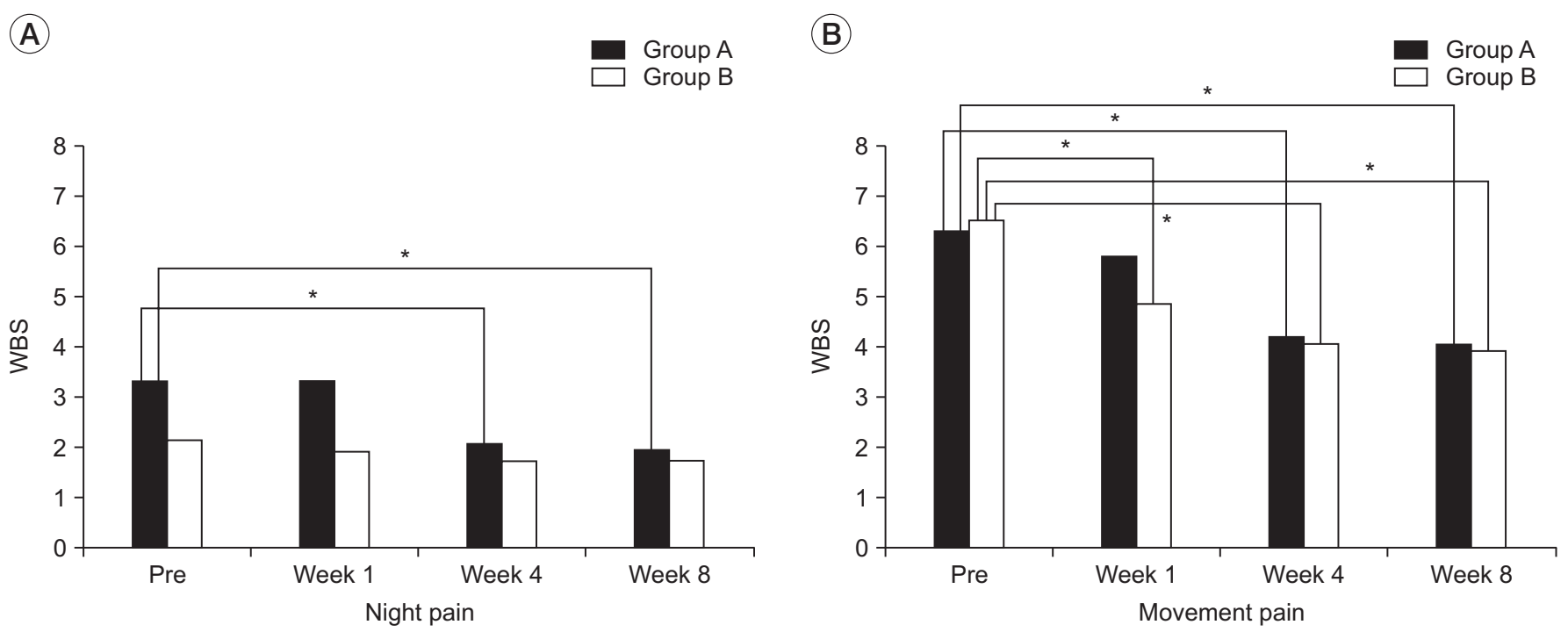

Fig. 3. Pain improvement at 1, 4, and 8 weeks after the intra-articular injection in the two groups (PP analysis, $\left.{ }^{*} \mathrm{p}<0.016\right)$. Post hoc testing showed no statistical differences between the two groups over time $(\mathrm{p}>0.05)$. WBS, WongBaker Scale; PP analysis, per protocol analysis.

week) or movement pain ( $\mathrm{p}=0.711$ at 8 th week) after 8 weeks (Fig. 3).

\section{DISCUSSION}

HSP usually develops after stroke, with $35 \%$ of cases of HSP developing within 2 weeks after stroke, and $72 \%$ of cases developing within 3 months after stroke [19]. HSP often causes difficulties, not only in activities of daily living but also in the effect of rehabilitation programs performed after stroke. It also negatively affects in functional recovery, and is associated with pain-induced depression and secondary complications of the shoulder $[7,20]$.

HSP is clinically very similar to adhesive capsulitis. It is known that muscle contracture develops due to constant immobility in hemiplegic patients, thus is accompanied by adhesive capsulitis [21]. A recent study of 106 HSP patients reported, that $56.6 \%$ had adhesive capsulitis, $17.9 \%$ shoulder subluxation, $13.2 \%$ complex regional pain syndrome, and $12.6 \%$ central pain [3]. Previous studies also reported that the main cause of HSP is adhesive capsulitis [22]. Another study reported a significant correlation between pain, limited ROM in hemiplegic shoulder and adhesive capsulitis [20]. Unfortunately, adhesive capsulitis may be the last stage due to chronic inflammation, injury and immobility in untreated HSP patients [23]. The earlier adhesive capsulitis is diagnosed in HSP patients, the more likely it is that treatment can help prevent longterm pain and stiffness.

Minor synovitis including edema, vascular dilation, and monocyte precipitation has been found in some patients with adhesive capsulitis, and steroid injection treatment is the therapy used with the expectation of an antiinflammatory effect [24]. Because fibrosis in the joint capsule plays the most important role in the inflammatory reaction, anti-inflammatory treatment is necessary to stop the fibrosis and improve the symptoms. When the effects of steroid injection dose and frequency in IAS treatment on shoulder pain were compared to the effects of steroid injection in combination with physical therapy in previous studies, no study reported that steroid treatment only would have better effects than other treatments.

Among the non-surgical treatments applicable to most stroke patients with HSP, oral analgesics and nonsteroidal anti-inflammatory drugs are not fully effective, and the risk of developing adverse effects from long-term drug use is high, especially in the elderly $[25,26]$. Also, the steroid used in IAS treatment in HSP patients has a usage limit because of the possibility of systemic and local adverse effects [6].

Hyaluronic acid is a long chain of polysaccharides composed of N-acetyl glucosamine and glucuronic acid. This forms the framework of proteoglycans and enables aggre- 
gation in the extracellular matrix; thus, it is an essential component in the structure and function of joint cartilage. The presence of hyaluronic acid in the joint cavity reduces the coefficient of friction and directly increases synovial fluid viscosity and elasticity. This provides a buffering effect for compressive and shearing forces and, has a role in protecting a joint. IAHA has been used in adhesive capsulitis due to its therapeutic effects such as reduced synovitis, influence on osmotic pressure, cartilage protective effect, prevention of intra-articular adhesions, and enhancement of the characteristics of synovial fluid [27].

According to the multicenter study performed by Blaine et al. [28], hyaluronic acid was relatively effective for the treatment of osteoarthritis that was not responsive to other non-surgical treatment. However, in the comparative study performed by Calis et al. [29], IAHA was not effective in patients with adhesive capsulitis compared to physical therapy or IAS. According to a recently conducted randomized controlled trial, IAHA had no additional effect on pain, ROM, and quality of life in patients with adhesive capsulitis who had already received physical therapy [30]. Another study showed that IAHA and IAS treatments gave significant improvement in passive ROM and a visual analog scale [16]. However, failure to accurately inject the joint cavity could not be excluded because the injection was performed in a blind manner in these previous studies. The accuracy of intra-articular injection in a blind manner is only $70 \%$ however, US guidance improves the accuracy of joint injection [31]. All of the injections in this study were performed using a posterior US-guided approach and injection accuracy was verified by checking the joint cavity expansion.

Many researchers have reported a positive effect of hydraulic distension in shoulder pain in non-stroke patients [32-35]. Thus, this study was designed to control any effect of hydraulic distension by injecting the same volume in all subjects, thus comparing only the pharmacological effects of hyaluronic acid and steroid.

In our study, shoulder pain and ROM improved significantly in both the IAHA and IAS groups. In the steroid group, it effectively controlled pain, especially during the early phase, which is probably due to the anti-inflammatory effect of the steroid. However, there was no great difference in the improvement of ROM or movement pain after 4 weeks of treatment in the IAHA or IAS group. For night pain, only the IAHA group had a statistically significant effect indicating that hyaluronic acid had a sufficient analgesic and anti-inflammatory effect compared to the steroid.

The significant of this study is that IAHA effectively controls HSP and improves ROM, and its efficacy does not differ from IAS at the 8th week of treatment. In other words, hyaluronic acid can be used when the steroid cannot be used due to concerns of diabetes and infection risk. Despite the treatment being administered to stroke patients in the acute and sub-acute phases of HSP, no adverse reactions of hyaluronic acid were observed.

One limitation of this study is that long-term clinical effects were not investigated due to the 8-week observational period. To overcome this limitation, a future study with long-term follow-up and observation after IAHA and IAS would be necessary. Second, the worsening of HSP is associated with the development and increase in spasticity after stroke. The early stage of this study was performed only in patients with minor spasticity of the upper extremities, so a study that compares the effect of treatment on spasticity as well as the effect of hyaluronic acid is needed. Third, although several confounding factors were controlled including medication and intensity of physical therapies, multiple factors might still have induced the pain of the patients including thalamic pain, insomnia and systemic diseases. The initial NSAIDs prescription for all subjects would also bring about the improvement of shoulder pain in HSP patients.

Nevertheless, our study could be meaningful as the first study to evaluate the efficacy of the hyaluronic acid injection compared to the steroid injection on poststroke HSP. Further study should be followed with more samples and controlling other confounding factors which could affect pain.

In conclusion, IAHA is less potent than IAS for reducing shoulder pain in stroke patients with HSP in the early period. However, there was no statistically significant difference after 4 weeks post-injection between the two groups. Based on the results of this study, IAHA could be an alternative treatment for managing HSP when the use of steroid is limited.

\section{CONFLICT OF INTEREST}

No potential conflict of interest relevant to this article 
was reported.

\section{ACKNOWLEDGMENTS}

This study was supported by a 2015 research grant from Pusan National University Yangsan Hospital.

\section{REFERENCES}

1. Griffin JW. Hemiplegic shoulder pain. Phys Ther 1986; 66:1884-93.

2. Jensen EM. The hemiplegic shoulder. Scand J Rehabil Med Suppl 1980;7:113-9.

3. Zhu Y, Su B, Li N, Jin H. Pain management of hemiplegic shoulder pain post stroke in patients from Nanjing, China. Neural Regen Res 2013;8:2389-98.

4. Vuagnat H, Chantraine A. Shoulder pain in hemiplegia revisited: contribution of functional electrical stimulation and other therapies. J Rehabil Med 2003; 35:49-54.

5. Pong YP, Wang LY, Wang L, Leong CP, Huang YC, Chen YK. Sonography of the shoulder in hemiplegic patients undergoing rehabilitation after a recent stroke. J Clin Ultrasound 2009;37:199-205.

6. Snels IA, Beckerman H, Twisk JW, Dekker JH, Peter De Koning, Koppe PA, et al. Effect of triamcinolone acetonide injections on hemiplegic shoulder pain: a randomized clinical trial. Stroke 2000;31:2396-401.

7. Gamble GE, Barberan E, Laasch HU, Bowsher D, Tyrrell PJ, Jones AK. Poststroke shoulder pain: a prospective study of the association and risk factors in 152 patients from a consecutive cohort of 205 patients presenting with stroke. Eur J Pain 2002;6:467-74.

8. Andersen LT. Shoulder pain in hemiplegia. Am J Occup Ther 1985;39:11-9.

9. Gaffney K, Ledingham J, Perry JD. Intra-articular triamcinolone hexacetonide in knee osteoarthritis: factors influencing the clinical response. Ann Rheum Dis 1995;54:379-81.

10. Gottlieb NL, Riskin WG. Complications of local corticosteroid injections. JAMA 1980;243:1547-8.

11. Pelletier JP, Martel-Pelletier J. Protective effects of corticosteroids on cartilage lesions and osteophyte formation in the Pond-Nuki dog model of osteoarthritis. Arthritis Rheum 1989;32:181-93.

12. Steinberg JJ, Sledge CB. Synovial factors and chondro- cyte-mediated breakdown of cartilage: inhibition by hydrocortisone. J Orthop Res 1983;1:13-21.

13. Nakazawa F, Matsuno H, Yudoh K, Watanabe Y, Katayama R, Kimura T. Corticosteroid treatment induces chondrocyte apoptosis in an experimental arthritis model and in chondrocyte cultures. Clin Exp Rheumatol 2002;20:773-81.

14. Hulmes DJ, Marsden ME, Strachan RK, Harvey RE, McInnes N, Gardner DL. Intra-articular hyaluronate in experimental rabbit osteoarthritis can prevent changes in cartilage proteoglycan content. Osteoarthr Cartil 2004;12:232-8.

15. Kitoh Y, Katsuramaki T, Tanaka H, Tanaka M, Kitabayashi N, Kataoka M, et al. Effect of SL-1010 (sodium hyaluronate with high molecular weight) on experimental osteoarthritis induced by intra-articularly applied papain in rabbits. Nihon Yakurigaku Zasshi 1992;100:67-76.

16. Kim HW, Kim HS, Ahn KH. The effect of intraarticular injection of hyaluronic acid and steroid in adhesive capsulitis of shoulder. J Korean Acad Rehabil Med 1999;23:117-23.

17. Peng PW, Cheng P. Ultrasound-guided interventional procedures in pain medicine: a review of anatomy, sonoanatomy, and procedures. Part III: shoulder. Reg Anesth Pain Med 2011;36:592-605.

18. Dogan SK, Ay S, Oztuna D, Aytur YK, Evcik D. The utility of the Faces Pain Scale in the assessment of shoulder pain in Turkish stroke patients: its relation with quality of life and psychologic status. Int J Rehabil Res 2010;33:363-7.

19. Bender L, McKenna K. Hemiplegic shoulder pain: defining the problem and its management. Disabil Rehabil 2001;23:698-705.

20. Bruckner FE, Nye CJ. A prospective study of adhesive capsulitis of the shoulder ("frozen shoulder') in a high risk population. Q J Med 1981;50:191-204.

21. Murie-Fernandez M, Carmona Iragui M, Gnanakumar V, Meyer M, Foley N, Teasell R. Painful hemiplegic shoulder in stroke patients: causes and management. Neurologia 2012;27:234-44.

22. Rizk TE, Christopher RP, Pinals RS, Salazar JE, Higgins C. Arthrographic studies in painful hemiplegic shoulders. Arch Phys Med Rehabil 1984;65:254-6.

23. Joynt RL. The source of shoulder pain in hemiplegia. Arch Phys Med Rehabil 1992;73:409-13. 
24. Neviser JS. Adhesive capsulitis of the shoulder: a study of the pathological findings in periarthritis of the shoulder. J Bone Joint Surg Am 1945;27:211-22.

25. Recommendations for the medical management of osteoarthritis of the hip and knee: 2000 update. American College of Rheumatology Subcommittee on Osteoarthritis Guidelines. Arthritis Rheum 2000;43:190515.

26. Singh G. Recent considerations in nonsteroidal antiinflammatory drug gastropathy. Am J Med 1998; 105:31S-8S.

27. Harris JD, Griesser MJ, Copelan A, Jones GL. Treatment of adhesive capsulitis with intra-articular hyaluronate: a systematic review. Int J Shoulder Surg 2011; 5:31-7.

28. Blaine T, Moskowitz R, Udell J, Skyhar M, Levin R, Friedlander J, et al. Treatment of persistent shoulder pain with sodium hyaluronate: a randomized, controlled trial. A multicenter study. J Bone Joint Surg Am 2008;90:970-9.

29. Calis M, Demir H, Ulker S, Kirnap M, Duygulu F, Calis HT. Is intraarticular sodium hyaluronate injection an alternative treatment in patients with adhesive capsulitis? Rheumatol Int 2006;26:536-40.
30. Hsieh LF, Hsu WC, Lin YJ, Chang HL, Chen CC, Huang V. Addition of intra-articular hyaluronate injection to physical therapy program produces no extra benefits in patients with adhesive capsulitis of the shoulder: a randomized controlled trial. Arch Phys Med Rehabil 2012;93:957-64.

31. Yamakado K. The targeting accuracy of subacromial injection to the shoulder: an arthrographic evaluation. Arthroscopy 2002;18:887-91.

32. Mulcahy KA, Baxter AD, Oni OO, Finlay D. The value of shoulder distension arthrography with intraarticular injection of steroid and local anaesthetic: a followup study. Br J Radiol 1994;67:263-6.

33. Sharma RK, Bajekal RA, Bhan S. Frozen shoulder syndrome. A comparison of hydraulic distension and manipulation. Int Orthop 1993;17:275-8.

34. van Royen BJ, Pavlov PW. Treatment of frozen shoulder by distension and manipulation under local anaesthesia. Int Orthop 1996;20:207-10.

35. Wybier M, Parlier-Cuau C, Baque MC, Champsaur P, Haddad A, Laredo JD. Distension Arthrography in Frozen Shoulder Syndrome. Semin Musculoskelet Radiol 1997;1:251-6. 